\title{
Chemical and Thermoelectric Properties of Hot Pressed and Spark Plasma Sintered Type-I Clathrate $\mathrm{Ba}_{8} \mathrm{Cu}_{4.8} \mathrm{Si}_{41.2}$
}

\author{
X. YAN, ${ }^{1,4}$ S. POPULOH ${ }^{2}$ A. WEIDENKAFF, ${ }^{2}$ P. ROGL, ${ }^{3}$ \\ and S. PASCHEN ${ }^{1,5}$ \\ 1.-Institute of Solid State Physics, Vienna University of Technology, Wiedner Hauptstr. 8-10, \\ 1040 Vienna, Austria. 2.-Laboratory for Solid State Chemistry and Catalysis, EMPA -Swiss \\ Federal Laboratories for Materials Science and Technology, Uberlandstrasse 129, 8600 Düben- \\ dorf, Switzerland. 3.-Institute of Physical Chemistry, Vienna University, Währingerstr. 42, 1090 \\ Vienna, Austria. 4.—e-mail: yanxinlin13@hotmail.com. 5.—e-mail: paschen@ifp.tuwien.ac.at
}

Nanostructuring has been considered as an effective way to reduce the thermal conductivity and enhance the thermoelectric performance in different material systems. Here, we present the chemical and thermoelectric properties of the nanostructured bulk type-I clathrate $\mathrm{Ba}_{8} \mathrm{Cu}_{4.8} \mathrm{Si}_{41.2}$. The samples were prepared by consolidating ball-milled nanopowders either by hot pressing or by spark plasma sintering. Fine powders and high sintering temperatures are needed to reach a high bulk density and high thermoelectric performance in the sintered samples. The highest ZT of 0.3 at $870 \mathrm{~K}$ is achieved in the most dense sample sintered at $800^{\circ} \mathrm{C}$ by hot pressing. Further improvement is expected if smaller grain sizes could be stabilized.

Key words: Thermoelectric materials, clathrates, hot pressing, spark plasma sintering

\section{INTRODUCTION}

The globe energy crisis and environmental issues have renewed the interest in thermoelectric (TE) research. ${ }^{1-3}$ In recent years, much effort has been focused on nanostructured thermoelectric materials since the first confirmation that reducing the dimensions of materials to form nanowires or superlattices can enhance the TE performance, ${ }^{4-6}$ which is gauged by the dimensionless figure of merit $Z T=T S^{2} / \rho \kappa$, where $T, S, \rho$, and $\kappa$ are the absolute temperature, Seebeck coefficient, electrical resistivity, and thermal conductivity, respectively. To manufacture model systems such as nanowires and superlattices is a complicated and expensive process. The importance is thus not only limited to fundamental studies. Much attention has focused on the research of nanostructured bulk materials. ${ }^{7,8}$

In particular, the improved TE performance in nanostructured materials is mostly attributed to the reduced thermal conductivity, arising from the

(Received July 19, 2015; accepted November 11, 2015;

published online December 7, 2015) increased scattering of phonons. ${ }^{8,9}$ A large grain size distribution is suited for the different phonon wavelengths. ${ }^{10,11}$

Methods for nanostructuring involve bottom-up and top-down processes. Ball milling (BM) is a simple, economic, and efficient top-down method, ${ }^{12-14}$ used to nanostructure many bulk TE materials. In this process, nano-sized grains are achieved through accumulating dislocations produced by severe plastic deformation via continuous collisions of balls. ${ }^{12}$ The kinetic energy of the balls, the milling time, and other process parameters play vital roles on grain size and properties of the resulting powders, which further influence the powder consolidation in the sintering process.

It remains, however, challenging to prevent grain growth during powder consolidation. There are different methods for consolidation including pressureless sintering and pressure-assisted sintering such as hot pressing (HP), hot isostatic pressing, ultrahigh-pressure sintering, and spark plasma sintering (SPS). ${ }^{12-14}$ The most important process parameters for powder consolidation are temperature, duration, and pressure in the case of pressure 
assistance. The ultimate goal in all these methods is to obtain dense samples with small grains. However, to reach a high bulk density and a small grain size in sintered samples generally is a conflicting requirement, ${ }^{15}$ which can only be realized under certain special conditions. For instance, the SPS method using relatively low temperatures and short holding times can frequently prepare dense samples with nano-sized grains. ${ }^{16,17}$ Here, we present the chemical and TE properties of the type-I clathrate $\mathrm{Ba}_{8} \mathrm{Cu}_{4.8} \mathrm{Si}_{41.2}$, which is synthesized by ball milling polycrystalline annealed samples, and subsequent powder consolidation either by HP or SPS. The selection of the composition is based on our previous finding that in the clathrates of the $\mathrm{Ba}-\mathrm{Cu}-\mathrm{Si}$ system the highest $\mathrm{ZT}$ value is achieved in the sample with the highest solid solubility of $\mathrm{Cu} .^{18,19}$ The main goal is to explore the relation between the process parameters for HP and SPS and the chemical and TE properties of the samples.

\section{EXPERIMENTAL PROCESS}

Ten large samples (4 $\mathrm{g}$ each) with the nominal composition $\mathrm{Ba}_{8} \mathrm{Cu}_{4.8} \mathrm{Si}_{41.2}$ were prepared in a highfrequency induction furnace from high-purity

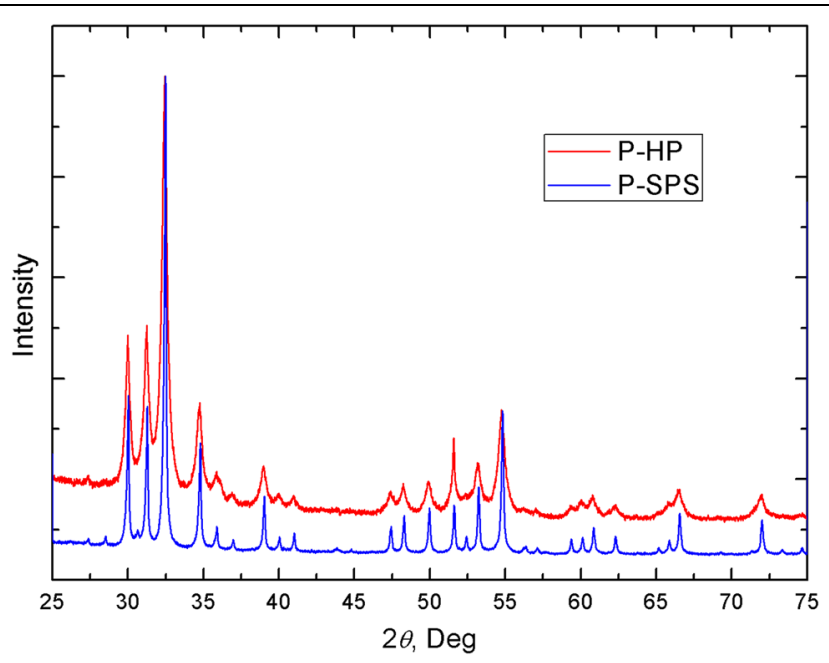

Fig. 1. XPD patterns for the powders of P-HP and P-SPS. The patterns were normalized to the highest peak of the clathrate phase. The weak peak at $\sim 28^{\circ}$ comes from the Si impurity phase. The main peaks can be indexed with the type-I clathrate phase. The peak width of P-HP is obviously broader than that of P-SPS. elements (99.96 wt.\% for $\mathrm{Ba}$, more than 99.999 wt.\% for $\mathrm{Cu}$ and $\mathrm{Si}$ ). The samples were annealed at $800^{\circ} \mathrm{C}$ for 10 days. The BM process was performed with two different setups: Setup 1, at the University of Vienna, the main process parameters are $120 \mathrm{rpm}$ for the speed of the main disk (low energy) and $13 \mathrm{~h}$ for the duration; setup 2, at the Laboratory for Solid State Chemistry and Catalysis in EMPA, the main process parameters are $400 \mathrm{rpm}$ for the speed of the main disk (high energy) and $1 \mathrm{~h}$ for the duration. The powders from setup 1 (denoted as P-HP) were then consolidated by HP in an FCT hot-press system with $T=680^{\circ} \mathrm{C}, 720^{\circ} \mathrm{C}, 760^{\circ} \mathrm{C}$, and $800^{\circ} \mathrm{C}, p=56 \mathrm{MPa}$ and the duration = $1 \mathrm{~h}$ in Ar. The powders from setup 2 (denoted as P-SPS) were compacted by SPS with $T=680^{\circ} \mathrm{C}, 720^{\circ} \mathrm{C}, 760^{\circ} \mathrm{C}$, and $820^{\circ} \mathrm{C}, p=50 \mathrm{MPa}$, and the duration $=10 \mathrm{~min}$ in Ar. The sintered samples are denoted as HP or SPS followed by the sintering temperature, e.g., HP680.

X-ray powder diffraction (XPD) data were collected using a HUBER-Guinier image plate system $(\mathrm{Cu}$ $\mathrm{K}_{\alpha 1}, 8^{\circ} \leq 2 \theta \leq 100^{\circ}$ ). Lattice parameters were calculated by least-squares fits to indexed $2 \theta$ values employing $\mathrm{Ge}$ as internal standard $\left(a_{\mathrm{Ge}}=\right.$ $0.5657906 \mathrm{~nm}$ ). The crystallite size and the residual strain were evaluated from the x-ray diffraction patterns using the MDI jade 6.0 software (Materials Data Inc., Liverpool, CA). Annealed $\left(300^{\circ} \mathrm{C}, 1\right.$ day) pure Si powder was used for the evaluation of the peak broadening due to the x-ray instrument. The density $D$ of the sintered samples was measured by the Archimedes method. The bulk density $D_{\mathrm{b}}$ is the ratio of $D / D_{\text {Theo, }}$, where $D_{\text {Theo }}$ is the theoretical density of the compound $\mathrm{Ba}_{8} \mathrm{Cu}_{4.8} \mathrm{Si}_{41.2}$.

The composition of the clathrate phase in the samples was determined by energy dispersive x-ray spectroscopy (EDX) in a scanning electron microscope (SEM) operated at $20 \mathrm{kV}$ (Zeiss Supra 55VP, probe size: $1 \mu \mathrm{m})$. In both series of sintered samples, the phase composition from EDX is $\mathrm{Ba}_{8} \mathrm{Cu}_{4.7} \mathrm{Si}_{41.3}$, with an error of 0.1 at./formula unit from the measurements.

The electrical resistivity and Seebeck coefficient were measured with a ZEM-3 (ULVAC-Riko, Japan) in a temperature range from room temperature to $600^{\circ} \mathrm{C}$. The thermal conductivity was calculated from the thermal diffusivity $D_{\mathrm{t}}$, measured by a laser flash method with a Flashline-3000 (ANTER, USA), the specific heat $C_{\mathrm{p}}$, and the density $D$ using the relation $\kappa=D_{\mathrm{t}} C_{\mathrm{p}} D . \quad C_{\mathrm{p}}$ were measured in a

Table I. Sample code, crystallite size $S_{\mathrm{c}}$, residual strain $\varepsilon$, and relative bulk density (the ratio between the measured density and the theoretical one) $D_{b}$ of sintered samples from the two series of HP and SPS

\begin{tabular}{|c|c|c|c|c|c|c|c|}
\hline Code & $\boldsymbol{S}_{\mathrm{c}}(\mathrm{nm})$ & $\varepsilon(\%)$ & $D_{\mathrm{b}}(\%)$ & Code & $S_{\mathrm{c}}(\mathrm{nm})$ & $\varepsilon(\%)$ & $D_{\mathrm{b}}(\%)$ \\
\hline HP680 & $60(4)$ & $0.036(3)$ & 77 & SPS680 & $135(4)$ & $0.034(3)$ & 78 \\
\hline HP720 & $78(2)$ & $0.028(3)$ & 83 & SPS720 & $335(2)$ & $0.028(3)$ & 80 \\
\hline HP760 & $80(2)$ & $0.009(3)$ & 92 & SPS760 & $>1000$ & $0.025(3)$ & 85 \\
\hline HP800 & $142(5)$ & $0.014(3)$ & 99 & SPS820 & $>1000$ & $0.032(3)$ & 89 \\
\hline
\end{tabular}




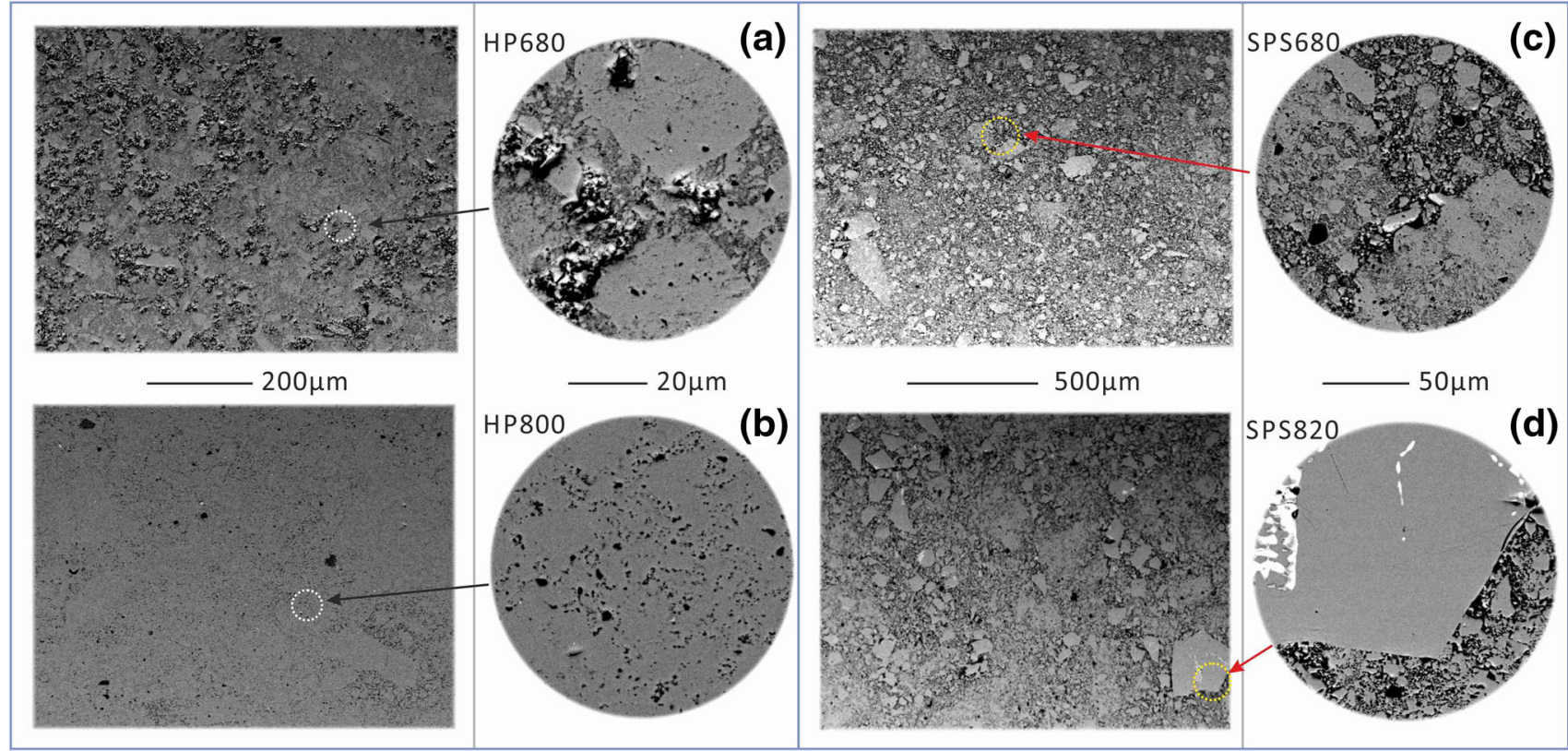

Fig. 2. Comparisons of microstructure for selected samples: (a) HP680, (b) HP800, (c) SPS680, and (d) SPS820. The particle size distribution in HP680 is narrower than that in SPS680, although the bulk density in both sample is similar. The porosity in HP800 is much smaller than that in SPS820, in line with the high bulk density in the former sample. A white phase is seen in both SPS680 and SPS820 samples.

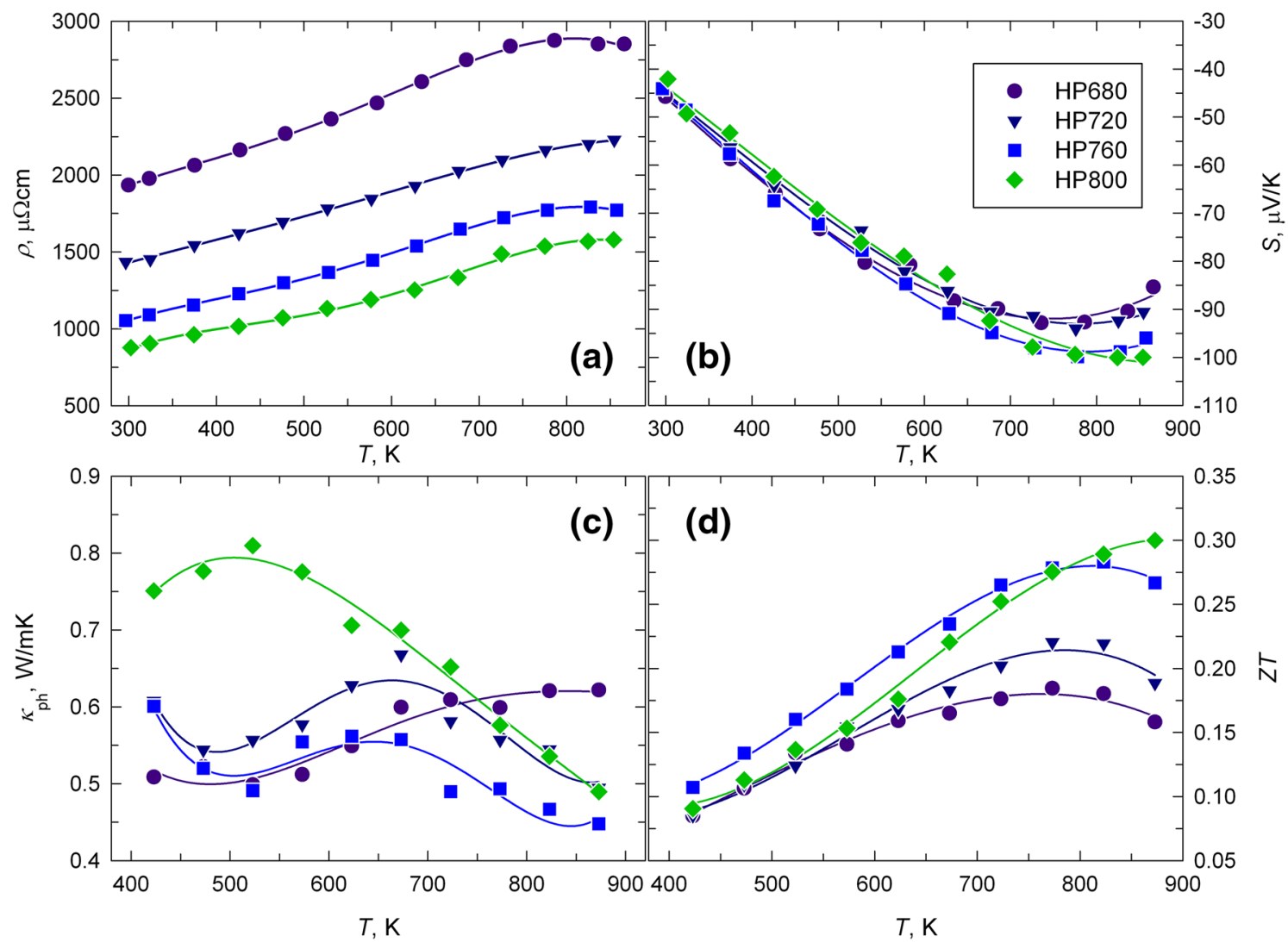

Fig. 3. Temperature dependence of the TE properties for the samples sintered by HP: (a) electrical resistivity $\rho(T)$; (b) Seebeck coefficient $S(T)$; (c) lattice thermal conductivity $\kappa_{\mathrm{ph}}(T)$; and (d) $Z T(T)$. 

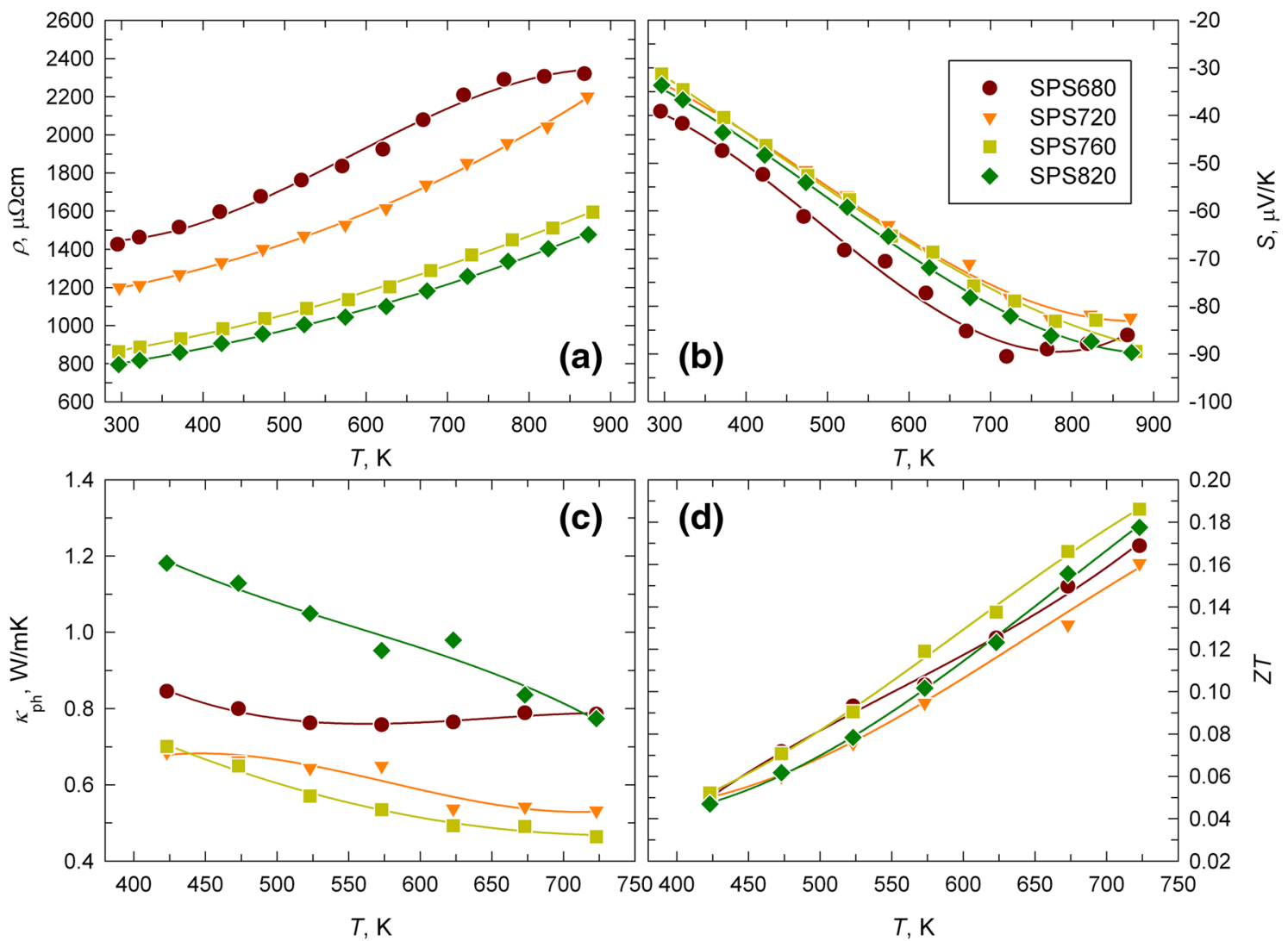

Fig. 4. Temperature dependence of the TE properties for the samples sintered by SPS: (a) electrical resistivity $\rho(T)$; (b) Seebeck coefficient $S(T)$; (c) lattice thermal conductivity $\kappa_{\mathrm{ph}}(T)$; and (d) $Z T(T)$.

comparative procedure in the Flashline-3000, using NIST steel as reference.

\section{RESULTS AND DISCUSSION}

XPD and EDX measurements revealed that all annealed samples are almost single type-I clathrate, with a minor Si impurity phase. The type-I clathrate is very stable during the BM process.

The crystallite size of as-milled powders depends on the process parameters for BM: The crystallite size for the P-HP powders is $25(2) \mathrm{nm}$, which is much smaller than the crystallite size $\sim 100 \mathrm{~nm}$ for the P-SPS powders, as evidenced by the broader peaks in the XPD pattern of the former powders (Fig. 1).

The crystallite size of sintered samples is listed in Table I. In both series of samples, the crystallite size and the relative bulk density increase with increasing sintering temperature. However, the sintering behavior is obviously related to the initial crystallite size of the powders. The powders from setup 1 with very fine grains can be easily sintered. A density close to the theoretical value and small and homogeneously distributed grains can be reached in the samples sintered by HP at high temperatures (Table I; Fig. 2). The crystallite size of the samples, sintered from the powders of setup 2 with coarse grains by SPS, is very large. Note that in the samples sintered by SPS, a spurious white phase is observed. This phase has the composition $\mathrm{Ba}_{5.8} \mathrm{Cu}_{58.0} \mathrm{Si}_{36.2}$ and the crystal structure type of $\mathrm{LaFe}_{9} \mathrm{Si}_{4}$ (space group $I 4 / \mathrm{mcm}$ ). ${ }^{18}$ The formation might be related to the strong local heating inherent in the SPS technique, which may lead to a local melting of the powders. Indeed, the amount of this phase increases with increasing sintering temperature.

Figures 3 and 4 summarize the results of our TE property measurements for both series of samples (HP and SPS), respectively. All samples show metallike behavior, with the electrical resistivity $\rho$ increasing with temperature. $\rho(T)$ decreases systematically with increasing sintering temperature in both series. The Seebeck coefficient $S$ in all samples is negative. $|S(T)|$ of the SPS samples is lower than that of the HP samples (Fig. 5). This might be either due to (i) a lower $\mathrm{Cu}$ content in the SPS samples, or to (ii) a strong trapping of charge carriers at the grain interface in the HP samples. Both reasons correspond to a lower charge carrier concentration in the HP samples $\left(S \propto 1 / n^{(2 / 3)}\right.$, see in Ref. 20).

The rationality for (i) is that the formation of the white phase with high $\mathrm{Cu}$ content naturally reduces the $\mathrm{Cu}$ content in the clathrate phase for element 


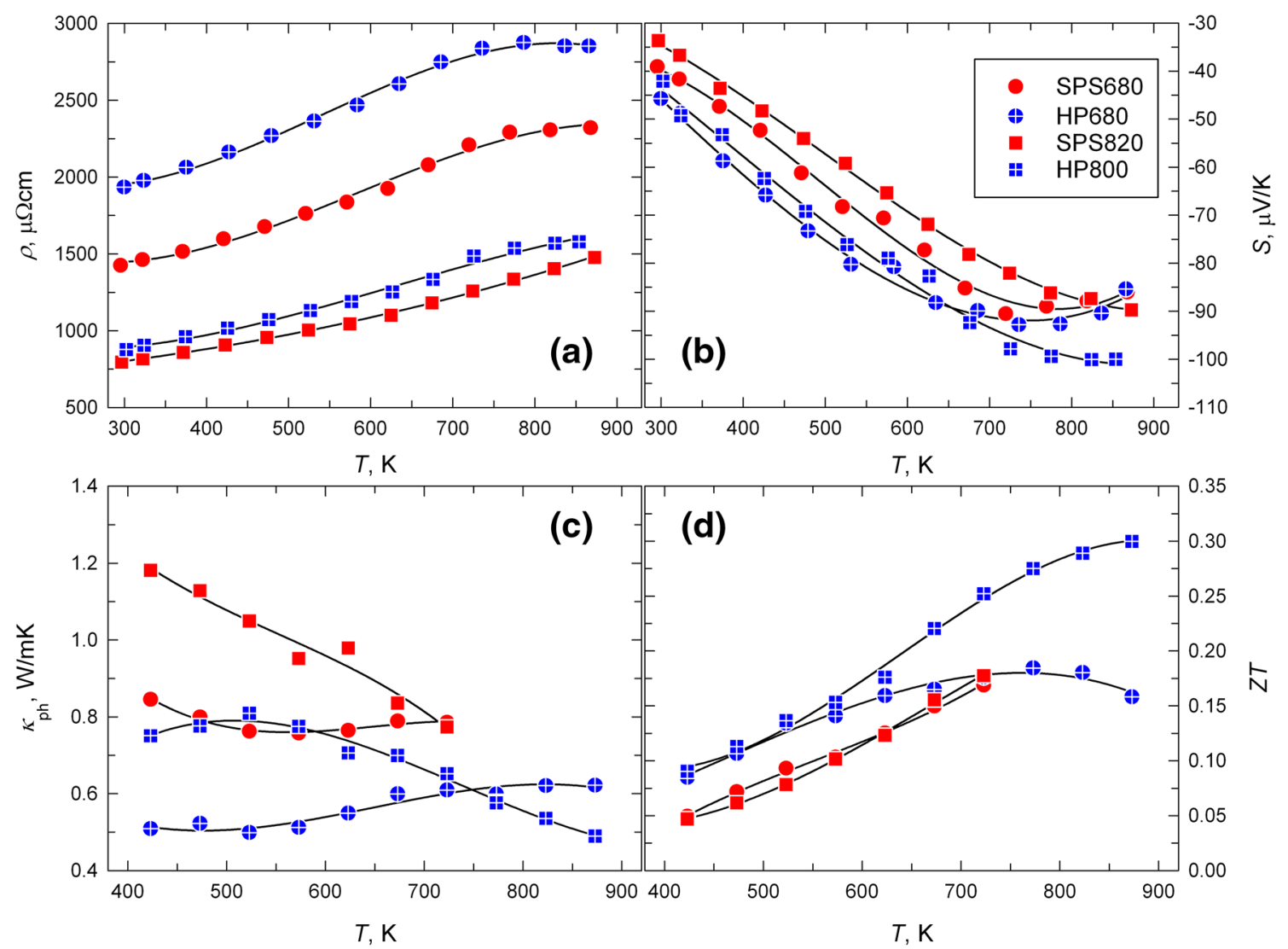

Fig. 5. Comparison of TE properties for the selected HPed and SPSed samples: (a) electrical resistivity $\rho(T)$; (b) Seebeck coefficient $S(T)$; (c) lattice thermal conductivity $\kappa_{\mathrm{ph}}(T)$; and (d) $Z T(T)$.

balance, although this reduction is below the detection limit of the EDX measurements. A higher charge carrier concentration is typically observed in samples with lower $\mathrm{Cu}$ content. ${ }^{19}$ This line of reasoning is further supported by the fact that the largest $|S(T)|$ is observed in the sample SPS680 with the smallest amount of the $\mathrm{Cu}$-rich impurity phase. As for (ii), it is well known that dangling bonds exist in grain boundaries. These bonds can trap electrons and thus reducing the charge carrier concentration for transport. ${ }^{20}$ The trapped electrons in boundaries further form local negative charge bands, which scatter electrons effectively and reduce the charge mobility. Since the grain size in the HP sample is smaller than that in the SPS samples, the interface density is larger and then more electrons can be trapped. In a related sample series, evidence for this electron trapping at grain boundaries was found from Hall effect measurements. $^{21}$

The lattice thermal conductivity $\kappa_{\mathrm{ph}}$ was calculated by subtracting the electronic contribution $\kappa_{\mathrm{e}}$ from the total thermal conductivity. $\kappa_{\mathrm{e}}$ was calculated from the electrical resistivity by the Wiedemann-Franz law with $L=2.44 \times 10^{-8} \mathrm{~W} \Omega \mathrm{K}^{-2}$. $\kappa_{\mathrm{ph}}(T)$ is very low for both series of samples (Figs. 3 and 4c). No systematic dependence on sintering temperature is observed. Overall, $\kappa_{\mathrm{ph}}(T)$ is lower for the HP samples than for the SPS samples, in agreement with the smaller grain sizes of the HP samples (Fig. 5c).

$Z T(T)$ is higher for the HP samples than for the SPS samples. It is mainly attributed to the higher Seebeck coefficient and lower thermal conductivity. The highest ZT of 0.3 at $870 \mathrm{~K}$ is reached in the HP800 sample, which has the highest bulk density.

\section{CONCLUSION}

In summary, we have presented the chemical and thermoelectric properties of the type-I clathrate $\mathrm{Ba}_{8} \mathrm{Cu}_{4.8} \mathrm{Si}_{41.2}$ fabricated from as cast and annealed samples by ball milling and hot pressing or spark plasma sintering. Our results indicated that fine powders and high sintering temperature are mandatory to obtain samples of $\mathrm{Ba}_{8} \mathrm{Cu}_{4.8} \mathrm{Si}_{41.2}$ with high bulk density and small grain sizes, and thus high TE performance. During the spark plasma sintering process, a spurious $\mathrm{Cu}$-rich phase forms, which might be held responsible for the decreased Seebeck coefficient in the SPS samples by reducing the $\mathrm{Cu}$ content of the main type-I clathrate. The lattice thermal conductivity is distinctly lower for samples with smaller grain sizes. This suggests that 
it could be further reduced if smaller grain sizes could be stabilized. The highest ZT of 0.3 at $870 \mathrm{~K}$ is reached in the densest sample.

\section{ACKNOWLEDGEMENTS}

The authors would like to thank F. Falmbigl and A. Grytsiv for hot pressing and M. Waas for the SEM/EDX investigation. This work was supported by the Austrian Science Fund (FWF Project TRP 176-N22) and the German Science fund (DFG SPP 1386 Project nanOcla).

\section{OPEN ACCESS}

This article is distributed under the terms of the Creative Commons Attribution 4.0 International License (http://creativecommons.org/licenses/by/4.0/ ), which permits unrestricted use, distribution, and reproduction in any medium, provided you give appropriate credit to the original author(s) and the source, provide a link to the Creative Commons license, and indicate if changes were made.

\section{REFERENCES}

1. F.J. DiSalvo, Science 285, 703 (1999).

2. B.C. Sales, Science 295, 1248 (2002).

3. T.M. Tritt, Science 283, 804 (1999).

4. R. Venkatasubramanian, E. Siivola, T. Colpitts, and B. O'Quinn, Nature 413, 597 (2001).

5. T.C. Harman, P.J. Taylor, M.P. Walsh, and B.E. LaForge, Science 297, 2229 (2002).
6. K.F. Hsu, S. Loo, F. Guo, W. Chen, J.S. Dyck, C. Uher, T. Hogan, E.K. Polychroniadis, and M.G. Kanatzidis, Science 303, 818 (2004).

7. G. Snyder and E. Toberer, Nat. Mater. 7, 105 (2008).

8. A. Minnich, M. Dresselhaus, Z. Ren, and G. Chen, Energy Environ. Sci. 2, 466 (2009).

9. C.J. Vineis, A. Shakouri, A. Majumdar, and M.G. Kanatzidis, Adv. Mater. 22, 3970 (2010).

10. J. Szczech, J. Higgins, and S. Jin, J. Mater. Chem. 21, 4037 (2011).

11. Z.G. Chen, G. Han, L. Yang, L. Cheng, and J. Zou, Prog. Nat. Sci. 22, 535 (2012).

12. C.C. Koch, Rev. Adv. Mater. Sci. 5, 91 (2003).

13. H.J. Fecht and Y. Ivanisenko, Nanostructured Materials: Processing, Properties, and Applications (Norwich: William Andrew Publishing, 2006), p. 119.

14. J.R. Groza, Nanostructured Materials: Processing, Properties, and Applications (Norwich: William Andrew Publishing, 2006), p. 173.

15. I. Zalite, P. Angerer, L.G. Yu, and K.A. Khor, I.O.P. Conf. Ser. Mater. Sci. Eng 23, 012039 (2011).

16. D. Kenfaui, G. Bonnefont, D. Chateigner, G. Fantozzi, M. Gomina, and J.G. Noudem, Mater. Res. Bull. 45, 1240 (2010).

17. Z. Munir, U. Anselmi-Tamburini, and M. Ohyanagi, J. Mater. Sci. 41, 763 (2006).

18. X. Yan, G. Giester, E. Bauer, P. Rogl, and S. Paschen, J. Electron. Mater. 39, 1634 (2010).

19. X. Yan, M.X. Chen, S. Laumann, E. Bauer, P. Rogl, R. Podloucky, and S. Paschen, Phys. Rev. B 85, 165127 (2012).

20. F.J. Blatt, Physics of Electronic Conduction in Solids (New York: McGraw-Hill, 1968), p. 180.

21. X. Yan, M. Falmbigl, G. Rogl, A. Grytsiv, A. Prokofiev, E Bauer, P. Rogl, M. Zehetbauer, and S. Paschen, J. Electron. Mater. 42, 1330 (2013). 